\title{
Desirability to characterize process capability
}

\author{
J. Jessenberger and C. Weihs ${ }^{\star}$
}

University of Dortmund ${ }^{\star \star}$

Department of Statistics

44221 Dortmund, Germany

\begin{abstract}
Over the past few years continuously new process capability indices have been developed, most of them with the aim to add some feature missed in former process capability indices. Thus, for nearly any thinkable situation now a special index exists which makes choosing a certain index as difficult as interpreting and comparing index values correctly.

In this paper we propose the use of the expected value of a certain type of function, the so-called desirability function, to assess the capability of a process. The resulting index may be used analogously to the classical indices related to $C_{p}$, but can be adapted to nearly any process and any specification. It even allows a comparison between different processes regardless of their distribution and may be extended straightforwardly to multivariate scenarios. Furthermore, its properties compare favorably to the properties of the "classical" indices.
\end{abstract}

\section{Introduction}

The amount of indices developed in recent years has lead to insecurity among the practitioners which index to use. The deficits of the classical indices related to the $C_{p}$-family have been extensively discussed (Kotz and Johnson (1993), Jessenberger (1999)) and have led to the continuous development of new indices which are custom-designed to eliminate these deficits one after the other. A special problem arises with the interpretation of all these index values. Most often the index values are associated with the percentage of conforming or non-conforming (NC) product. However, this is only true if implicit assumptions are valid. A $C_{p}$-value of 1 will only indicate a percentage of $\mathrm{NC}$ product of $0.27 \%$ if and only if the specification is symmetric, the process is normally distributed, the mean equals the specification midpoint and the process is under statistical control. In practice, the validity of these assumptions often is not verified. If one additionally takes into account that most capability values are estimates rather than true values and that the

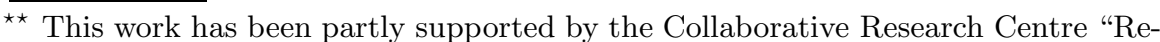
duction of Complexity in Multivariate Data Structures" (SFB 475) of the German Research Foundation (DFG). The simulations were run on personal computers using the software S-PLUS 4.3 (Statistical Sciences Inc., Seattle). The simulation programs are available through the first author.

* e-mail: weihs@statistik.uni-dortmund.de 
estimators of nearly all process capability indices are biased it is understandable that some authors propose to stop using process capability indices at all (Pignatiello and Ramberg (1993)).

However, in this paper we will propose another index which overcomes the above-mentioned problems and has the properties needed to assess the quality of a process: ease of interpretation, validity for all specification types, flexibility with respect to process distributions, and existence of a good estimator.

In the following we will present the new index for uni- and multivariate processes, and compare its properties with the main classical indices in the bivariate case. As all classical indices are dependent on the validity of the normal assumption we will only discuss the new index for normal processes although the extension for other distributions is straightforward. For quality characteristics $X$ we will assume a univariate or multivariate normal distribution (with p dimensions) with mean (vector) $\mu$ and variance $\sigma^{2} /$ covariance matrix $\Sigma$, denoted by $X \sim \mathrm{N}\left(\mu, \sigma^{2}\right)$ and $X \sim \mathrm{Np}(\mu, \Sigma)$, respectively. In the multivariate case often a process ellipsoid is used to characterize the process properties. This is defined to be the ellipsoid which contains a certain percentage (usually 99.73\%) of the process distribution. In the univariate case the ellipsoid collapses to an interval containing the desired percentage of the process distribution.

For the univariate case, specifications consist of a target value $T$ and lower and/or upper specification limits (LSL, USL). If the target value lies on the midpoint $m:=(L S L+U S L) / 2$ of the specification interval, the specification is called a (two-sided) symmetric specification, else a (two-sided) asymmetric specification. If either the lower or the upper specification limit is infinite while still retaining the nominal optimal target value, the specification is called one-sided. Multivariate specification is described by the Cartesian product of the univariate specifications and denoted by (M1). Frequently, ellipsoids are used as multivariate specification regions which are typically the largest-volume ellipsoids completely contained in (M1), denoted by (M2). In this paper we will develop uni- and multivariate indices, but restrict ourselves to the discussion of the multivariate case because of space restrictions. For comparison we will use the most common classical multivariate indices. A multivariate analogue of the univariate $C_{p}$-Index (Taam et al. (1993)) is given by:

$\mathrm{MVC}_{p}:=\frac{\operatorname{vol}(\text { max. vol. ellipsoid in specification })}{\operatorname{vol}(\text { process ellipsoid })}=\left(\frac{|A|}{|\Sigma|}\right)^{1 / 2}\left(\frac{1}{\chi_{p ; 0.9973}^{2}}\right)^{p / 2}$.

$\chi_{p ; 0.9973}^{2}$ denotes the $99.73 \%$-quantile of the $\chi^{2}$-distribution with $\mathrm{p}$ degrees of freedom and $A=\operatorname{diag}\left(d_{1}^{2}, \ldots, d_{p}^{2}\right), d_{j}:=\left(U S L_{j}-L S L_{j}\right) / 2, j=1, \ldots, p$, is the specification matrix defining the specification ellipsoid (M2) given by $\left\{x \mid(x-m)^{\prime} A^{-1}(x-m) \leq 1\right\}, m:=\left(m_{1} \ldots m_{p}\right)^{\prime}, m_{i}:=\left(U S L_{i}+L S L_{i}\right) / 2$. The multivariate analogue of the $C_{p m}$-Index (Taam et al. (1993)) additionally 
includes the Mahalanobis distance between the mean and the target vector to measure a possible deviation of the mean from the target:

$$
\begin{aligned}
\operatorname{MVC}_{p m}:= & \frac{\operatorname{vol}(\max . \text { vol. ellipsoid in specification })}{\operatorname{vol}\left((x-T)^{\prime} \Sigma_{T}^{-1}(x-T) \leq \chi_{p ; 0.9973}^{2}\right)} \\
& \text { where } \quad \Sigma_{T}:=E\left[(X-T)(X-T)^{\prime}\right]=\Sigma+(\mu-T)(\mu-T)^{\prime} \\
= & \left(\frac{|A|}{|\Sigma|}\right)^{1 / 2}\left(\frac{1}{\chi_{p ; 0.9973}^{2}}\right)^{p / 2} / \sqrt{1+(\mu-T)^{\prime} \Sigma^{-1}(\mu-T)} .
\end{aligned}
$$

\section{Combining capability and desirability - the indices EDU and EDM}

Desirability indices were invented in experimental design to summarize several response variables and thus identify the direction of optimization (Derringer and Suich (1980)). Typically, several - possibly contradicting - response variables have to be optimized simultaneously where each response can be modeled as a (different) function of a common set of predictors. The aim is to have each response approach as much as possible their target optimum value while at the same time ensuring that the overall result still is unacceptable if only one of the responses attains an unacceptable value.

Derringer and Suich (1980) propose to transform the responses to so-called "desirability values" between 0 and 1 , which takes the value 1 if the quality characteristic attains the target value and decreases if it deviates from the target. Undesirable values have the desirability 0. Typically, for a two-sided specification with target $\mathrm{T}$ and lower and upper specification limits LSL and USL one would choose a desirability function as follows:

$$
\begin{aligned}
& D_{r ; s}: \mathrm{IR} \rightarrow[0,1], \\
& x \mapsto D_{r ; s}(x):= \begin{cases}(x-L S L)^{r} /(T-L S L)^{r} & , \text { for } x \in[L S L, T] \\
(U S L-x)^{s} /(U S L-T)^{s} & , \text { for } x \in[T, U S L] \\
0 & , \text { else. }\end{cases}
\end{aligned}
$$

where $r, s \in \mathrm{IR}$ are suitably chosen constants to reflect how rapidly a deviation from the target becomes undesirable. For one-sided specifications the idea is easily extended. Usually in one-sided specifications there exists a point beyond which desirability improves only marginally and thus is defined to be constant 1 .

The desirability index is then defined as the geometric mean of the desirability functions in each dimension (cf. Harrington (1965), and Derringer and Suich (1980)). In this paper we will use a different approach and define the indices EDU and EDM as the expected desirability for a given process. The EDU-Index (expected desirability, univariate) is defined as:

$$
E D U:=E(D(X))
$$


Analogously, the multivariate index EDM (expected desirability, multivariate) is given as

$$
E D M:=E\left(D_{M V}(X)\right),
$$

where $D(x)$ and $D_{M V}(x)$ are suitable univariate and multivariate desirability functions.

This construction has the advantage that the EDU and EDM index values in principle can be calculated regardless of the distribution of the process and regardless of the specification, as long as the expectation over the desirability function exists.

Through the custom-designed desirability function the practitioner gains flexibility as any shape and structure of the specification region can be modeled through this function. Taking the expectation even allows differently distributed processes to be compared directly with each other whereas for the classical indices normality must hold.

The explicit form of the EDU-Index with a linear desirability function $D_{1}$ for a normal distribution is given in the following (cf. Jessenberger (1999)) as an example.

Let $X \sim \mathrm{N}\left(\mu, \sigma^{2}\right)$ with density function $f$ and distribution function $F$, $(L S L, T, U S L)$ a two-sided specification and $D_{1}$ the linear desirability function $(r=s=1)$.

Let $\delta:=(\mu-T) / d, \eta:=\sigma / d, \beta:=(T-m) / d, d:=(U S L-L S L) / 2, m:=$ $(U S L+L S L) / 2, a:=(-(\delta+\beta)-1) / \eta, b:=(-(\delta+\beta)+1) / \eta$. Then EDU is given as:

$$
\begin{aligned}
E D U= & \delta\left[\frac{2}{1-\beta^{2}} \Phi\left(\frac{-\delta}{\eta}\right)-\frac{\Phi(a)}{1+\beta}-\frac{\Phi(b)}{1-\beta}\right] \\
& -\eta\left[\frac{2}{1-\beta^{2}} \varphi\left(\frac{-\delta}{\eta}\right)-\frac{\varphi(a)}{1+\beta}-\frac{\varphi(b)}{1-\beta}\right]-\Phi(a)+\Phi(b)
\end{aligned}
$$

where $\varphi$ and $\Phi$ denote the standard normal density and distribution function, respectively.

The multivariate desirability function $D_{M V}$ is defined as follows:

$$
D_{M V}\left(x_{1}, x_{2}, \ldots, x_{p}\right):=\min \left(D_{1}\left(x_{1}\right), D_{2}\left(x_{2}\right), \ldots, D_{p}\left(x_{p}\right)\right),
$$

where $D_{i}\left(x_{i}\right)$ are desirability functions for $X_{i}, i=1, \ldots, p$.

This also shows an obvious way of finding the distribution of $D_{M V}$. Thus, $D_{M V}$ is defined as a non-standard desirability index in that the univariate desirability functions are joined via a minimum function and not via the more usual geometrical mean (cp. Kim and Lin (2000)). For the explicit expression of EDM in the bivariate case with linear desirability functions and normality see Jessenberger (1999).

In this paper we will concentrate on the comparison of EDM with the classical indices. 


\section{Discussion}

Bivariate normal processes will be used to illustrate the performance of the EDM-Index. Table 1 gives the variances $\sigma_{1}^{2}=\sigma_{2}^{2}$, covariances $\sigma_{12}$ and correlations $\rho$ of the examined processes $\mathrm{A}, \mathrm{B}, \mathrm{C}$ and $\mathrm{D}$. The two quality characteristics of the processes $\mathrm{A}$ and $\mathrm{C}$ are highly correlated, whereas the variables for processes B and D are uncorrelated.

For both quality characteristics a symmetric univariate specification of

\begin{tabular}{|c|c|c|c|c|}
\hline \hline Process & $\mathrm{A}$ & $\mathrm{B}$ & $\mathrm{C}$ & $\mathrm{D}$ \\
\hline$\sigma_{1}^{2}=\sigma_{2}^{2}$ & 50 & 15 & 15 & 10 \\
$\sigma_{12}$ & 49 & 0 & 14 & 0 \\
$\rho$ & 0.98 & 0 & 0.9333 & 0 \\
\hline \hline
\end{tabular}

Table 1. Example processes

$(L S L, T, U S L)=(35,50,65)$ is assumed. The Cartesian product of the univariate specifications will be denoted by $(\mathrm{M} 1)=(35,50,65) \times(35,50,65)$, the specification given by the largest-volume ellipsoid will be denoted by (M2). Furthermore, the behavior for on-target and off-target processes will be examined. For on-target processes $\mu$ equals the target value: $\mu=T=(50,50)^{\prime}$ , for off-target processes the process mean is moved into the direction of the bottom-left corner of the specification area: $\mu=(40,40)^{\prime} \neq T$. Figure 1 shows process and specification ellipses for on- and off-target comparison. Table 2

\begin{tabular}{||l||l|l|l||l|l|l||}
\hline \hline Process & $\begin{array}{l}1-q \\
(\mu=T)\end{array}$ & $M V C_{p}$ & EDM & $1-q$ & $M V C_{p m}$ & EDM \\
\hline \hline $\mathrm{A}$ & 0.175 & 1.912 & 0.595 & 0.171 & 1.100 & $\mathbf{0 . 3 3 8}$ \\
$\mathrm{B}$ & 0.466 & 1.268 & 0.709 & 0.340 & 0.335 & 0.211 \\
$\mathrm{C}$ & 0.612 & $\mathbf{3 . 5 3 2}$ & 0.760 & $\mathbf{0 . 5 1 3}$ & $\mathbf{1 . 2 5 7}$ & 0.311 \\
$\mathrm{D}$ & $\mathbf{0 . 7 5 1}$ & 1.902 & $\mathbf{0 . 7 6 2}$ & 0.470 & 0.415 & 0.224 \\
\hline \hline
\end{tabular}

Table 2. Example processes

shows the percentage of conforming product and the process capability index (PCI) values for $M V C_{p}, M V C_{p m}$ and EDM for the four example processes, the highest values indicating the best processes are marked in bold type. For all indices except EDM the processes $\mathrm{C}$ or D are the best. This result seems to be intuitively sensible because these are the processes with the smallest variation. For $M V C_{p}$ and $M V C_{p m}$ even the ranking of the processes A to $\mathrm{D}$ is the same: The best process is the process with the smallest variation and highest correlation (process $\mathrm{C}$ ) and the worst process is the process with largest variation and without correlation (process $\mathrm{B}$ ). The reason for the good performance of process $\mathrm{C}$ is that the volume of the corresponding process ellipsoid is much smaller than the specification ellipsoid. However, especially in the case of process A (ranked second for both classical indices) this ignores 
the fact that a high percentage of the product produced with process A lies outside the specification limits and is thus not or only partially fit for use. The process ranking according to the EDM index is different. For the ontarget case the ranking of the processes by the EDM index is analogous to the percentage of conforming product 1-q. Thus process D is the best and process A the worst process according to EDM. For the off-target case the situation changes. The highly correlated processes $\mathrm{A}$ and $\mathrm{C}$ are preferred to the processes without correlation (B and D). It is intuitively clear that this is because the deviation of the process means coincides with the direction of correlation for processes $\mathrm{A}$ and $\mathrm{C}$ so that a larger percentage of the distribution is close to the target. With the same argument $\mathrm{A}$ is preferred to $\mathrm{C}$. Overall it can be said that the EDM index - per definition -prefers processes that are "on average close to target".
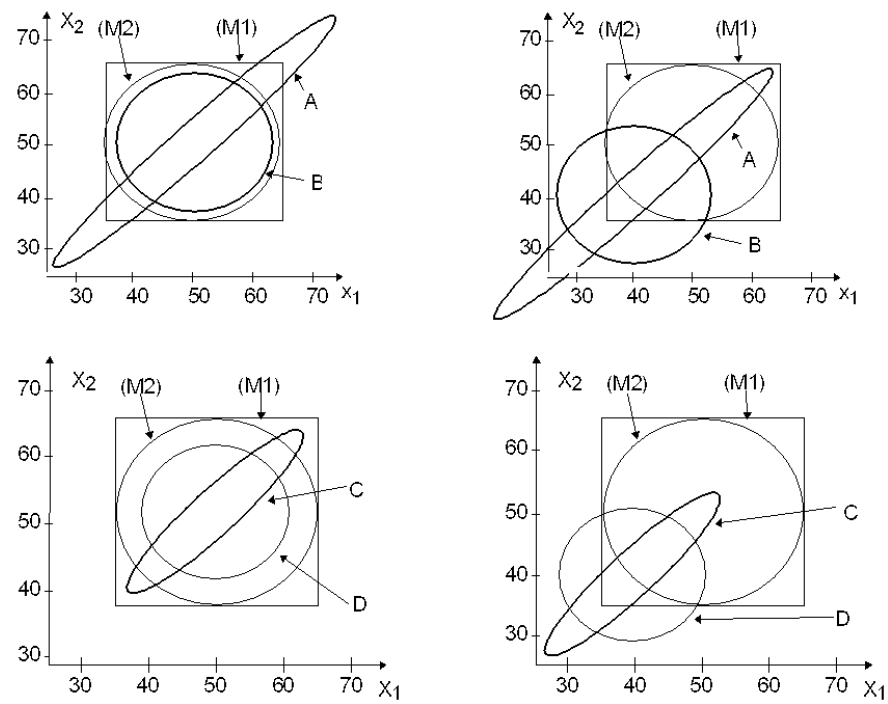

Fig. 1. Specification (M1), process and specification ellipses (M2) for bivariate processes

\section{Estimation}

Let us concentrate again on the bivariate case. Let $\delta:=D(\mu-T), H:=D \Sigma D$ and $\beta:=D(T-m)$, where $D:=\operatorname{diag}\left(1 / d_{1}, 1 / d_{2}\right), m:=\left(m_{1} m_{2}\right)^{\prime}, m_{i}:=$ $\left(U S L_{i}+L S L_{i}\right) / 2$ and $d_{i}:=\left(U S L_{i}-L S L_{i}\right) / 2, i=1,2$. With this, $M V C_{p}$, $M V C_{p m}$ may be written as functions of $\delta, \eta$ or $H$, respectively, EDU was expressed in analogous terms above.

For the estimation of $M V C_{p}$ and $M V C_{p m}$ estimates of the transformed mean and variation are inserted into the functional form and the resulting value 
is used as an estimate for each index. For the estimation of the EDM-Index two approaches are considered. If the functional form of EDM is known, it is possible to insert estimates instead of the unknown distribution parameters expectation and variation (plug-in estimator). Secondly, estimates can be achieved by the average of individually determined desirability values:

Let $X, X_{i}:=\left(X_{1 i}, X_{2 i}\right)^{\prime} \sim \mathrm{N}_{2}(\delta, H)$ and $X, X_{1}, \ldots, X_{n}$ independently identically distributed variables. Further, let $\hat{\delta}:=\left(\hat{\delta}_{1}, \hat{\delta}_{2}\right)^{\prime}$ with $\hat{\delta}_{j}:=\left(X_{j 1}, \ldots, X_{j n}\right) /$ $n, j=1,2$, and $\hat{\eta}_{j}^{2}:=\frac{1}{n-1} \sum_{i=1}^{n}\left(X_{j i}-\hat{\delta}_{j}\right)^{2}, \hat{\eta}_{12}^{2}:=\frac{1}{n-1} \sum_{i=1}^{n}\left(X_{1 i}-\hat{\delta}_{1}\right)\left(X_{2 i}-\hat{\delta}_{2}\right)$, $\hat{\rho}=\hat{\delta}_{12} /\left(\hat{\eta}_{1} \hat{\eta}_{2}\right)$.

Then two possible estimators of the EDM-Index are given as:

1. $\operatorname{EDM}:=\operatorname{EDM}\left(\hat{\delta}_{1}, \hat{\delta}_{2}, \hat{\eta}_{1}, \hat{\eta}_{2}, \hat{\rho}\right)$ and

2. DิMV $:=\frac{1}{n} \sum_{i=1}^{n} D_{M V}\left(X_{1 i}, X_{2 i}\right)$.

From the functional form of the estimator it is clear that it is unbiased and asymptotically normally distributed.

\section{Simulation}

A common criterion to compare the performance of estimators is the mean squared error MSE. However, the MSE is heavily scale-dependent. As the values of the "classical" indices are not bounded from above as is EDU/EDM, to compare the performance of the estimators for EDU/EDM with the estimators for the "classical" indices it is necessary to standardize the MSE by the magnitude of the estimated quantity:

Let $\operatorname{MSE}(\hat{\theta})$ denote the mean squared error of the estimator $\hat{\theta}$ for a statistic $\theta>0$ or $\theta<0$. Then the standardized MSE, $\operatorname{MSE}_{s t}(\hat{\theta})$, is defined as $\operatorname{MSE}_{s t}(\hat{\theta}):=\operatorname{MSE}(\hat{\theta}) / \theta^{2}$.

For the bivariate case combinations of the values $-0.5,0,0.5$ for $\beta_{1}, \beta_{2}, \delta_{1}, \delta_{2}$ and $\rho$ and combinations of the values 0.1 and 1.1 for $\eta_{1}$ and $\eta_{2}$ have been examined. The number of random variates used for estimating the location and variation parameters is $\mathrm{n}=50$, the number of repetitions $\mathrm{N}=1000$ which was sufficient for a good precision of the simulation (cf. Jessenberger (1999)). Due to space restrictions we give summary results rather than all detailed results which can be found in the above-mentioned literature. EDMM and DMV were shown to be better than $\mathrm{MVC}_{p m}$ in terms of the maximal values of $\mathrm{MSE}_{s t}$. Moreover, the $\mathrm{MSE}_{s t}$ for EDM and $\hat{\mathrm{DMV}}$ are maximal for small values of the distribution parameters. If the variation and correlation increases the mean standardized error decreases and even the maximum $\mathrm{MSE}_{s t}$ is reduced by half or more. In contrast, for $\mathrm{MVC}_{p m}$ the maximum value of $\mathrm{MSE}_{s t}$ may be attained throughout all considered combinations of $\eta_{1}, \eta_{2}$ and $\rho$. Only the spread of the values decreases. With regard to the comparison between EDMM and $\mathrm{DMV}$, the former has smaller $\mathrm{MSE}_{s t}$ than DMV for all simulations. Thus overall, EDM is the best estimator among the estimators considered. 


\section{Conclusion}

In this paper we have presented a new index for assessing process capability which is based on the expected value of desirability functions. These desirability functions assign a "desirability value" to each value a quality/process characteristic may take. An average desirability of a process may then be used as a measure for process capability.

The proposed approach is feasible for any given specification and distribution and allows a wide range of processes to be compared directly. A comparison of the newly proposed EDM index with the classical multivariate analoga of $C_{p}$ and $C_{p m}$ shows that the new index compares favorably. Moreover, in choosing different desirability functions EDU/EDM-indices offer a good chance to reflect virtually every specification region as long as the corresponding expectation exists. Furthermore, regardless of underlying process distribution the interpretation of the index values is always the same so that processes following different distributions may be compared directly. Simulation studies show that an obvious estimator for EDM exhibits equally good or better behavior than the usual estimators for the classical indices.

\section{References}

DERRINGER, G.C. and SUICH, R. (1980): Simultaneous optimization of several response variables. Journal of Quality Technology, 12, 337-45.214-219.

HARRINGTON JR., E.C. (1965): The desirability function. Industrial Quality Control 21, 494-498.

JESSENBERGER, J. (1999): Prozeßfähigkeitsindizes in der Qualitätssicherung. Ph.D. Thesis, Department of Statistics, University of Dortmund.

KIM K.-J. and LIN, D.K.J. (2000): Simultaneous optimization of mechanical properties of steel by maximizing desirability functions. Applied Statistics 49(3), 311-326.

KOTZ, S, and JOHNSON, N.L. (1993): Process Capability Indices. Chapman \& Hall, London.

PIGNATELLO, J.J. and RAMBERG, J.S. (1993): Process Capability Indices: Just say "No!". ASQC Quality Congress Transactions, 92-104, Boston.

TAAM, W., SUBBAIAH, P. and LIDDY, J.W. (1993): A note on multivariate capability indices. Journal of Applied Statistics 20(3), 229-351. 